\title{
VARIANTES ANATÓMICAS DE LA ARTERIA HEPÁTICA COMÚN
}

\author{
José D. Fonseca Figueroa ${ }^{1}$, Valeria L. Alvarado Rodríguez ${ }^{1}$, Pedro Estrada \\ McDermott $^{1}$, Verónica Morales Vindas ${ }^{1}$, Kevin Leiva Gabriel ${ }^{2}$, Jéssica González \\ Fernández ${ }^{2}$
}

\author{
${ }^{2}$ Departamento de Anatomía, Escuela de Medicina Universidad de Costa Rica. San José, \\ Costa Rica
}

\begin{abstract}
RESUMEN
Introducción: Las variantes de la arteria hepática común $(\mathrm{AHC})$ han sido estudiadas y descritas desde la antigüedad, con la subsecuente aparición de clasificaciones anatómicas para su correcta identificación. Se ha descrito que hasta un $40 \%$ de la población presentan dichas variantes. Metodología: Se realizó una revisión sistemática de la literatura utilizando los descriptores "arteria hepática", "variación anatómica" y "anatomía" donde se obtuvo un registro de 22203 que habían sido reportados por diferentes investigadores anteriormente y se analizaron en conjunto. Resultados: La variantes anatómicas de la AHC fueron estudiadas y clasificadas por Michels, Lippert y Pabst, y Hiatt. Según la clasificación de Hiatt, $80,46 \%$ de los individuos se agrupan dentro del grupo I, $6,46 \%$ dentro del grupo II, $6,10 \%$ en el grupo III, $1,50 \%$ en el grupo IV, $1,32 \%$ en el grupo $V$ y $0,02 \%$ en el grupo VI. El grupo X de Michels, no contemplado en la clasificación de Hiatt representó el 0,04\% de la muestra. Conclusiones: Se recomienda realizar estudios de imagen preoperatorios cuando se considere apropiado puesto que podría incidir en la disminución de complicaciones quirúrgicas y postquirúrgicas y se debería considerar realizar estudios descriptivos para determinar la prevalencia de variantes anatómicas en la población local.
\end{abstract}

Palabras Clave: Arteria hepática; Variación anatómica; Anatomía. Fuente: MeSH

\footnotetext{
ABSTRACT

Introduction: Variants of the common hepatic artery have been studied and described since ancient times, with the subsequent appearance of anatomical classifications for their correct identification. It has been reported that up to $40 \%$ of the population present these variants. Methodology: A systematic review of the literature was carried out by using the key words "hepatic artery", "anatomic variation" and "anatomy",
}

resulting in a registry of 22203 individuals previously reported by various researchers which was analyzed as a whole. Results: Anatomical variants were studied and described by Michels, Lippert \& Pabst, and Hiatt. According to the Hiatt classification, $80,46 \%$ of individuals were sorted in group I, $6,46 \%$ in group II, $6,10 \%$ in group III, $1,50 \%$ in group IV, $1,32 \%$ in group $\mathrm{V}$ and $0,02 \%$ in group VI. Michels' group $\mathrm{X}$, not included in Hiatt's classification, represented $0,04 \%$ of the sample. Conclusions: Preoperative imaging studies are recommended when appropriate since it could result in a decrease in surgical and post-surgical complication. Descriptive studies that determine the prevalence of anatomical variants in the local population should be considered.

Key Words: Hepatic artery; Anatomic variation; Anatomy. Source: MeSH.

\section{INTRODUCCIÓN}

El estudio descriptivo de la vasculatura hepática ha ocupado un lugar importante entre los anatomistas desde la antigüedad. Inicialmente, como lo menciona Ottone (2012), en 1756 Haller incorporó variaciones del tronco celíaco dentro de sus estudios. Por su parte, también menciona que Tidemann en 1822 y Adachi en 1928 realizaron estudios sobre variantes arteriales post-mortem (Ursic et al., 2007; Ottone et al., 2012).

\footnotetext{
* Correspondencia a: José D. Fonseca Figueroa. daniel.fon.fig.97@gmail.com
}

Recibido: 22 de Julio, 2020. Revisado: 7 de Agosto, 2020. Aceptado: 18 de Agosto, 2020. 
Con la incorporación de técnicas de inyección intravascular con resinas plásticas y acrílicas, para el siglo XX, Ottone (2012) menciona que cobran relevancia los análisis clásicos realizados por Flint en 1923, Michels en 1966 y Hiatt en 1994, los cuales fueron destacados debido a la clasificación macroscópica de las variantes que aportaron. Posteriormente, la aparición de las técnicas de diagnóstico por imagen sumó información para confeccionar un mapa vascular completo, tanto de la circulación extrahepática como intrahepática (Ursic et al., 2007; Ottone et al., 2012; Pidal et al., 2014).

\begin{tabular}{|c|c|c|}
\hline Clasificación & Total (n=22203) & Porcentaje \\
\hline Hiatt I & 17865 & $80,46 \%$ \\
\hline Hiatt II & 1434 & $6,46 \%$ \\
\hline Hiatt III & 1355 & $6,10 \%$ \\
\hline Hiatt IV & 334 & $1,50 \%$ \\
\hline Hiatt V & 293 & $1,32 \%$ \\
\hline Hiatt VI & 4 & $0,02 \%$ \\
\hline Michels X & 8 & $0,04 \%$ \\
\hline Otros & 45 & $0,20 \%$ \\
\hline No clasificados & 865 & $3,90 \%$ \\
\hline
\end{tabular}

Tabla I. Distribución de las variantes de la arteria hepática común (AHC) según casos reportados previamente en la literatura. (Fonseca et al., 2017; Gomes et al., 2014; Hiatt et al., 1994;Michels, 1966; Noussios et al., 2017; Ottone et al., 2012; Rodríguez y Fallas, 2011; Saiz et al., 2017;Skórzewska et al., 2014)

En la actualidad, se ha identificado que cerca del $40 \%$ de los pacientes presenta variantes anatómicas en la circulación hepática (Doherty, 2014). Omitir la presencia de estas, ha generado un aumento en complicaciones quirúrgicas tales como: aumento en el riesgo de lesión vascular intraoperatoria (que cause sangrado, isquemia o necrosis), disfunción hepática postoperatoria (con elevación de enzimas hepáticas), ligadura accidental de variantes anatómicas, fístulas anastomóticas e inclusive fugas biliares anastomóticas. Estas complicaciones generalmente causan un aumento en el tiempo quirúrgico, aumentan la tasa de transfusión y la cantidad de complicaciones postoperatorias (Doherty, 2014; Fonseca et al., 2017; Sitarz et al., 2018; Gkaragkounis et al., 2019).

Las variaciones en la vascularización son especialmente importantes en el contexto del trasplante de hígado, ya que, su identificación precisa es crucial para la arterialización efectiva y por lo tanto, el éxito del procedimiento en el receptor del órgano. Sin embargo, otros procedimientos como las angiografías también precisan del conocimiento detallado de las variantes pues este permite al radiólogo intervencionista disminuir el tiempo del procedimiento y evitar embolización de arterias sanas. Otros procedimientos donde se ha visto la disminución de complicaciones incluyen: cirugía hepatobiliar, cirugía pancreática, y cirugía gástrica (Doherty, 2014; Fonseca et al., 2017; Gkaragkounis et al., 2019).

En este trabajo se realizará una revisión sistemática acerca de las principales variantes anatómicas de la vascularización hepática y sus diferentes clasificaciones, reportadas en la literatura, con el fin de valorar la importancia de la identificación de estas en la práctica médicoquirúrgica.

\section{MATERIALES Y MÉTODO}

Se llevó a cabo una revisión sistemática de la literatura en Google Académico, PubMed, AccessMedicine y SciELO, utilizando como descriptores de búsqueda: "arteria hepática", "variación anatómica", "anatomía"; con los mismos términos para la búsqueda en inglés. Se estableció como periodo de búsqueda desde enero de 1966 hasta diciembre del 2019, esto con el objetivo de incluir documentos históricamente relevantes en el tema y a su vez, analizar publicaciones más recientes. El uso de los descriptores arrojó el siguiente número de documentos: 57800 de Google Académico, 5149582 de PubMed, 12897 de AccessMedicine, 4072 de SciELO. Dado que con estos descriptores se encuentra una gran cantidad de artículos y libros, se redujo la cantidad de documentos a analizar mediante el uso de dos o 
más descriptores simultáneos y otros términos relevantes: "variación anatómica de la arteria hepática común", "relevancia clínica de la arteria hepática común", "clasificación de Hiatt", "clasificación de Michels". Lo cual obtuvo como resultado: 34960 de Google Académico, 2228 de PubMed, 44 de AccessMedicine, 5 de SciELO. De estos últimos se hizo revisión por título y luego por resúmenes eliminando las redundancias considerando solamente los documentos relevantes para la revisión.

Los criterios de selección de la información clínica relevante utilizados incluyeron: 1) artículos o libros con información actualizada de las variantes anatómicas de la arteria hepática común, 2) aquellos que dentro de su metodología se incluyera el estudio de dichas variantes en cadáveres $\mathrm{y} / \mathrm{o}$ métodos de imágenes, 3) artículos con información respecto al uso de las clasificaciones en la práctica clínica, 4) artículos que incluyeran la relevancia de dichas clasificaciones en la práctica clínica. Finalmente, se procedió al análisis cualitativo del texto completo, con lo cual se recuperaron: 4 de Google Académico, 8 de PubMed, 3 de AccessMedicine, 5 de SciELO.

\section{RESULTADOS}

A partir de las fuentes empleadas para esta revisión, se obtuvieron registros de 22203 individuos que ya habían sido reportados por diferentes investigadores anteriormente y se analizaron en conjunto (Tabla I). Los diferentes estudios clasificaron los pacientes utilizando la clasificación de Michels o la de Hiatt. Para los efectos de esta revisión se utiliza mayoritariamente la clasificación de Hiatt puesto que esta clasificación agrupa distintos grupos de Michels, por lo que en los casos reportados únicamente con la clasificación de Hiatt no se puede conocer el grupo de Michels correspondiente, mas es posible determinar el grupo de Hiatt al que corresponden los casos reportados mediante la clasificación de Michels. Sin embargo, ya que la clasificación de Hiatt no incluye el grupo $X$ de Michels, esta se incluye como una variante por aparte.

Cabe destacar que algunos autores utilizaron únicamente la clasificación de Michels y otros únicamente la clasificación de Hiatt, por lo que se generó un subregistro de los grupos VI de Hiatt y $X$ de Michels.

La determinación de dichas variantes tiene aplicaciones en los campos de cirugía y radiología intervencionista (Michels, 1966; Hiatt et al., 1994; Noussios et al., 2017).

\section{DISCUSIÓN}

\section{Origen embriológico}

El origen de las variantes de la AHC se puede explicar desde el punto de vista embriológico. La mayor parte del desarrollo vascular ocurre durante el primer trimestre, de craneal a caudal. Durante la cuarta y quinta semana de gestación, las aortas dorsales (izquierda y derecha) se fusionan para formar la aorta abdominal. Posteriormente, durante el desarrollo de los intestinos, las arterias ventrales segmentarias se fusionan en la línea media formando el tronco celiaco (TC), la arteria mesentérica superior (AMS), y la arteria mesentérica inferior. Se ha descrito que un fallo en este proceso lleva a variaciones anatómicas del TC y sus ramas. (Ottone et al., 2012; White et al., 2015; Pinal et al., 2018)

Asimismo, se ha planteado que en la embriogénesis humana las arterias intestinales metaméricas primitivas están conectadas por una anastomosis longitudinal anterior $\mathrm{y}$, por lo tanto, la persistencia o desaparición de distintas porciones de este plexo arterial determina la aparición de numerosas variaciones del TC y la AMS. En el desarrollo fetal, los factores que pueden contribuir a dichos cambios en el plexo arterial incluyen: la rotación del intestino medio, la hernia fisiológica, la migración hacia la izquierda del bazo y los cambios hemodinámicos en las vísceras abdominales. También se ha propuesto la influencia de factores genéticos y étnicos; por su parte, no se ha encontrado que el género sea un factor relevante. (Ottone et al., 2012;Wang et al., 2014; White et al., 2015; Pinal et al., 2018)

\section{Anatomía topográfica convencional}

EI TC se origina de la aorta abdominal aproximadamente a nivel de vértebra T12, y tiene como ramas a la arteria gástrica izquierda (AGI), arteria esplénica (AE) y AHC. Normalmente la $\mathrm{AHC}$ se bifurca al atravesar el foramen omental, en la arteria gastroduodenal y la arteria hepática propia (AHP). La AHP asciende anterior a la vena porta y medial al conducto biliar común hasta que, cercano a su origen o cercano a la fisura portal principal, se divide en dos ramas: una izquierda y una derecha. (Busuttil y Klintmalm, 2015; Favelier et al., 2015; Brunicardi et al., 2019)

\section{Clasificación de las variantes de la AHC}

1.1. Clasificación de Michels

El estudio de las variantes anatómicas de la AHC guarda relevancia para la prevención y reducción de riesgos quirúrgicos producto de iatrogenia. $\mathrm{A}$ 
lo cual, Michels publica en 1966 su propia descripción de estas variantes, realizada mediante la disección de 200 pacientes cadavéricos, con el fin de reducir el riesgo de las cirugías abdominales. Aunque observa 26 variantes posibles de la circulación hepática, enfatiza los 10 grupos básicos más frecuentes. Esta clasificación ha sido clásicamente utilizada para describir las variantes de la vasculatura hepática. (Michels, 1966; Rodríguez y Fallas, 2011; Favelier et al., 2015; Fonseca et al., 2017) La clasificación es la siguiente (ver Fig. 1):

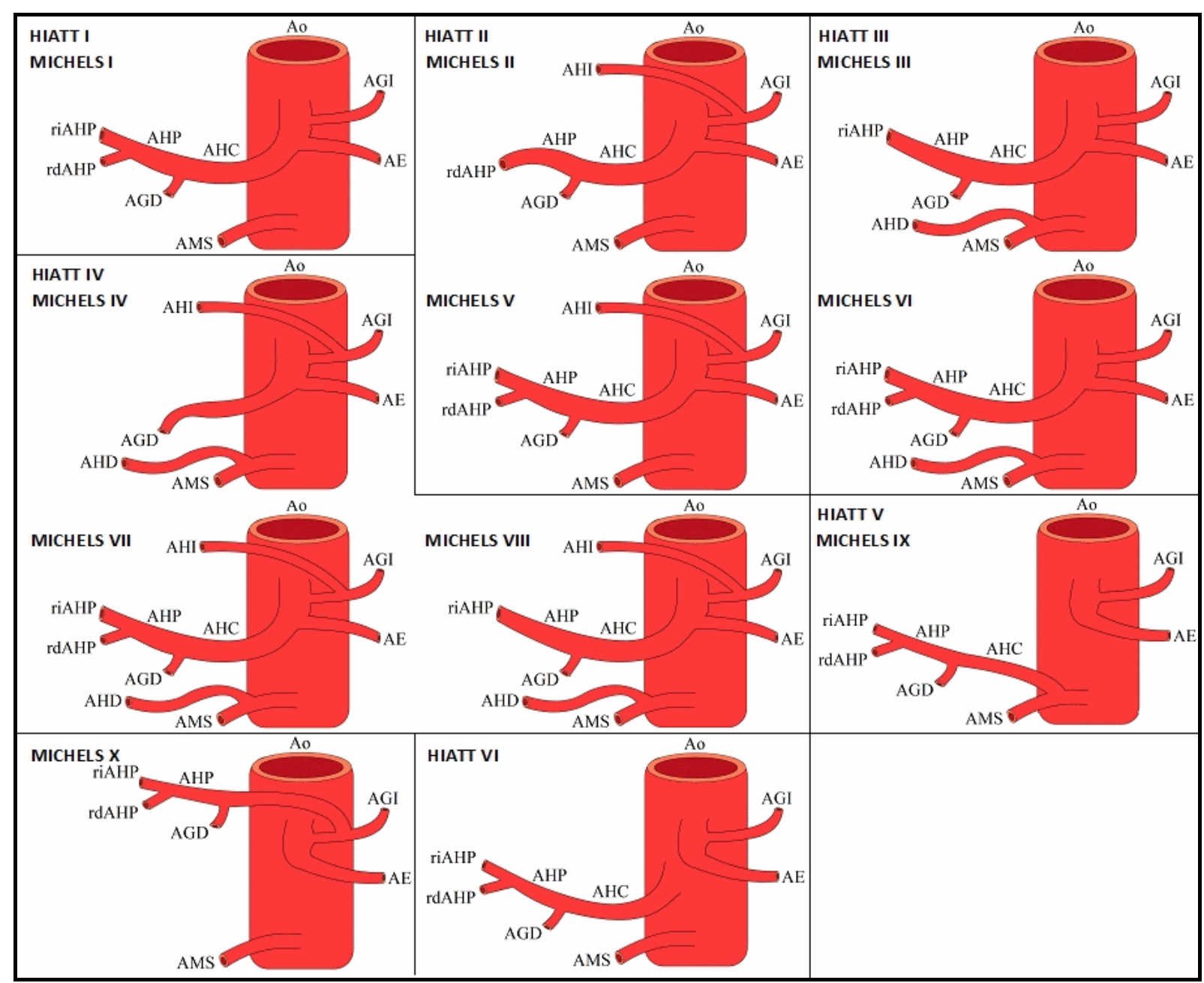

Figura 1. Representación de la anatomía descrita en las clasificaciones de Michels y Hiatt. (Favelier et al., 2015; Hiatt et al., 1994;Michels, 1966) Abreviaturas: AHC (arteria hepática común), AGD (arteria gastroduodenal), AHP (arteria hepática propia), riAHP (rama izquierda de la arteria hepática propia), rdAHP (rama derecha de la arteria hepática propia), AHI (arteria hepática izquierda), AHD (arteria hepática derecha), AGI (arteria gástrica izquierda), AE (arteria esplénica), AMS (arteria mesentérica superior).

- Grupo I: AHC se origina en TC, AHP da dos ramas: izquierda y derecha.

- Grupo II: AHC se origina en TC, AHP da rama derecha, arteria hepática izquierda (AHI) ectópica originada en la AGI.

- Grupo III: AHC se origina en TC, AHP da rama izquierda, arteria hepática derecha (AHD) ectópica originada en la AMS.

- Grupo IV: No hay AHC, AHD ectópica originada en la AMS, AHI ectópica originada en la AGI.
- Grupo V: AHC se origina en TC, AHP da dos ramas: izquierda y derecha, AHI accesoria originada en la AGI.

- Grupo VI: AHC se origina en TC, AHP da dos ramas: izquierda y derecha, AHD accesoria originada en la AMS.

- Grupo VII: AHC se origina en TC, AHP da dos ramas: izquierda y derecha, AHI accesoria originada en la AGI, AHD accesoria originada en la AMS. 
- Grupo VIII: AHC se origina en TC, AHP da rama izquierda, $\mathrm{AHI}$ accesoria originada en la AGI, AHD ectópica originada en la AMS.

- Grupo IX: AHC se origina en AMS, AHP da dos ramas: izquierda y derecha.

- Grupo X: AHC se origina en AGI, AHP da dos ramas: izquierda y derecha.

Sin embargo, la clasificación de Michels no incluía todas las variantes existentes. Con el advenimiento de técnicas de imagen como la angiografía, se han descrito al menos 5 variantes no contempladas en la clasificación de Michels. (Saiz et al., 2017)

\subsection{Clasificación de Lippert y Pabst}

Lippert y Pabst publicaron su libro "Arterial variations in man. Classification and Frequency" en 1985, incluyendo una sección sobre las variaciones anatómicas de la AHC. Se describen 15 variantes anatómicas, incluyendo la anatomía convencional, con su respectiva prevalencia. Sin embargo, las fuentes utilizadas en esta revisión referencian con mayor frecuencia las clasificaciones de Michels y Hiatt. (Lippert y Pabst, 1985)

\subsection{Clasificación de Hiatt}

Por otra parte, Hiatt y colaboradores realizaron su propio estudio de tipo observacional descriptivo en 1994, en el cual se tomó una muestra de 1000 pacientes donantes hepáticos vivos y se clasificó su anatomía según estaba descrita en los registros quirúrgicos, comparándola con la descrita por Michels. Decidieron simplificar su clasificación agrupando ciertas variantes de la clasificación original. Esta clasificación describe seis variantes y también se conoce también como clasificación de Michels modificada. Se considera la más representativa por tener la muestra más amplia. (Hiatt et al., 1994;Rodríguez y Fallas, 2011; Gomes et al., 2014)

La clasificación fue agrupada de esta manera (ver Fig. 1):

- Grupo I: Grupo I de la clasificación de Michels original.

- Grupo II: Grupo II ó V de la clasificación de Michels original.

- Grupo III: Grupo III ó VI de la clasificación de Michels original.

- Grupo IV: Grupo IV ó VII ú VIII de la clasificación de Michels original.

- Grupo V: Grupo IX de la clasificación de Michels original.

- Grupo VI: AHC se origina en la aorta, AHP da dos ramas: izquierda y derecha.

Hay dos diferencias principales entre las clasificaciones de Michels y Hiatt. La primera es que Hiatt agrupa las arterias de un origen común en un mismo grupo ya sean ectópicas, que dan circulación de tipo terminal a una porción del hígado, o accesorias, que dan circulación colateral a una porción del hígado junto con otra rama de la AHP. La segunda diferencia es que Hiatt omite de su clasificación el grupo $X$ de Michels, en el cual la AHC se origina de la AGI ya que no observó esta variante y agregó un grupo propio, el grupo VI que describe una AHC originada de la aorta. (Rodríguez y Fallas, 2011; Favelier et al., 2015; Fonseca et al., 2017; Noussios et al., 2017)

\section{Relevancia en la práctica clínica}

Michels y Hiatt desarrollaron sus respectivas clasificaciones de las variantes de la AHC con el propósito de disminuir los eventos adversos relacionados a iatrogenia durante procedimientos quirúrgicos. Michels explica que la irrigación completa del lóbulo hepático izquierdo puede comprometerse durante una gastrectomía en pacientes con las variantes II y IV de la clasificación original (aproximadamente el 11\% de la población) puesto que, si la AGI es ligada muy cerca de su origen, habrá necrosis en la zona irrigada por la $\mathrm{AHI}$, situación que es potencialmente mortal. (Michels, 1966; Hiatt et al., 1994)

Las variantes de la $\mathrm{AHC}$ pueden visualizarse de forma no invasiva mediante técnicas de imagen y esto tiene aplicaciones en el campo de la radiología intervencionista, y cirugía hepatobiliar, como lo son: (a) radiodiagnóstico de anatomía y patología hepatobiliar, (b) colocación de catéter para quimioterapia intraarterial, ya que requiere que el segmento hepático de interés presente circulación terminal o cauterizar las arterias colaterales, (c) radio o quimioembolización de la arteria nutricia de tumores hepáticos, tomando en cuenta la localización del mismo y la posible existencia de vascularización colateral y (d) cirugía de trasplante hepático, ya que se debe considerar las diferentes variantes anatómicas para planear la técnica quirúrgica y garantizar la adecuada irrigación del injerto. (Favelier et al., 2015)

Sin embargo, no en todos los casos se puede conocer la irrigación hepática previo a la cirugía, por lo que se recomienda identificar la totalidad de la irrigación hepática antes de ligar cualquier estructura arterial, ya que las variantes anatómicas pueden tener recorridos poco comunes. Noussios menciona reportes de caso de pacientes portadores de las variantes III o IX de Michels (aproximadamente el $13.5 \%$ de la población) sometidos a cirugía hepatobiliar que por compromiso de la circulación hepática proveniente de la AMS presentaron necrosis de la vía biliar con consecuente fuga de la 
anastomosis bilioentérica. (Michels, 1966; Noussios et al,. 2017)

\section{Conclusiones}

Se ha visto que hasta un $80 \%$ de la población posee la anatomía convencional (Hiatt I o Michels I) y alrededor de un 15\% alguna de las otras variantes anatómicas descritas tanto por Michels como por Hiatt. Esto hace de suma importancia que el personal médico que realice procedimientos médicos o quirúrgicos en los órganos irrigados por el TC tenga en cuenta estas variantes, por lo que se recomienda que se realice algún estudio de imagen o radiodiagnóstico para tener en cuenta la anatomía con la que se va a trabajar.

Asimismo, es recomendable que se estudie la prevalencia de las variantes de la población a tratar, ya sea mediante el estudio postmortem o por imágenes. Ya que, si bien es cierto, que hasta el $40 \%$ de la población en general presenta variaciones en su anatomía, esto podría variar geográficamente por factores genéticos $y$ étnicos, al igual que la incidencia de las diferentes variantes estudiadas. Por lo cual, los servicios de cirugía, especialmente la sección de trasplante hepático debería contar con esta información estadística.

Por su parte, hay que tener especial cuidado en las variantes II y IV de la clasificación de Michels cuando se va a realizar una gastrectomía, para no comprometer la irrigación hepática de la $\mathrm{AHI}$ y en las variantes III y IX de Michels, debido a que una intervención quirúrgica donde no se tengan los cuidados preoperatorios del estudio de estas variables, puede llevar a necrosis de la vía biliar.

Por lo anterior, los procedimientos que involucren la irrigación hepática sean diagnósticos o resolutivos, requieren el conocimiento adecuado de las variantes descritas para disminuir los eventos adversos y aumentar la probabilidad de éxito de estos.

\section{Conflicto de intereses}

No hay ningún conflicto de interés por declarar.

\section{Financiamiento}

Los autores no recibieron ningún financiamiento por o para la realización de este artículo.

\section{Aprobación ética \\ No corresponde.}

\section{Consentimiento informado}

No corresponde.

\section{Contribuciones}

VA: recolección de información, análisis de la bibliografía, escritura del manuscrito, elaboración de figura. PE: recolección de información, análisis de la bibliografía, escritura del manuscrito, elaboración de figura. JF: recolección de información, análisis de la bibliografía, escritura del manuscrito, elaboración de figura. VM: recolección de información, análisis de la bibliografía, escritura del manuscrito, elaboración de figura. KL: revisión del manuscrito, escritura del manuscrito. JG: revisión del manuscrito.

\section{AGRADECIMIENTOS}

Al personal del LaMoNec de la Universidad de Costa Rica por su guía y apoyo en el desarrollo de este proyecto e incentivar la realización de investigación por parte de los estudiantes; al Dr. Diego Pineda Martínez y la Dra. Olivia Espinosa Vázquez, docentes de la Universidad Nacional Autónoma de México, por su interés en nuestro trabajo, y sus recomendaciones.

\section{BIBLIOGRAFÍA}

Brunicardi F, Andersen D, Billiar T, Dunn D, Kao L, Hunter J, Matthews J, Pollock R. 2019. Schwartz's Principles of Surgery. 11va Edición, Nueva York: McGraw-Hill, pag: 1-2448.

Busuttil R, Klintmalm G. 2015. Transplantation of the liver. 3ra Edición, Filadelfia: Elsevier, pag: 1-1568.

Doherty G. 2014. CURRENT Diagnosis \& Treatment: Surgery. 14va Edición, Nueva York: McGraw-Hill, pag: 1-1392.

Favelier S, Germain T, Genson P, Cercueil J, Denys A, Krausé D, Guiu B. 2015. Anatomy of liver arteries for interventional radiology. DiagnInterv Imaging 96: 537-46. (Doi:10.1016/ j.diii.2013.12.001).

Fonseca O, Lima H, Rabelo P, Melo P, Amorim A, Lacerda C. 2017. Anatomic Variations of Hepatic Artery: A Study in 479 Liver Transplantations. ABCD Arq Bras Cir Dig 30: 35-37. (Doi: 10.1590/0102-6720201700010010) Gkaragkounis A, Fanariotis $M$, Tepetes K, Fezoulidis I, Vassiou K. 2019. Celiac Trunk and Hepatic Arteries: Anatomical Variations of Liver Arterial Supply as Detected with Multidetector Computed Tomography in 1,520 Patients and its Clinical Importance. Clin Anat. (Doi: 10.1002/ ca.23511).https://onlinelibrary.wiley.com/doi/10. 1002/ca.23511 (Último acceso 14 de junio de 2020) 
Gomes G, Encina C, Guerra F, Lira G, Fonseca $J$, Pardins J. 2014. Atypical origin of the common hepatic artery. ABCD Arq Bras Cir Dig 27: 308-309. Doi:10.1590/S0102-67202014 000400021

Hiatt J, Gabbay J, Busuttil R. 1994. Surgical Anatomy of the Hepatic Arteries in 1000 Cases. Ann Surg 220: 50-52. (Doi:10.1097/00000658199407000-00008)

Lippert H, Pabst R. 1985. Arterial variations in man. Classification and frequency. Berlin: Springer, pag: 30-45.

Michels N. 1966. Newer anatomy of the liver and its variant blood supply and collateral circulation. Am Jour Surg 112: 337-47. (Doi:10.1016/0002-9610(66)90201-7)

Noussios G, Dimitriou I, Chatzis I, Katsourakis A. 2017. The Main Anatomic Variations of the Hepatic Artery and Their Importance in Surgical Practice: Review of the Literature. J Clin Med Res 9: 248-52. (Doi:10.14740/jocmr2902w)

Ottone N, Blasi E, Dominguez M, Medan C. 2012. Tronco Celíaco Mesentérico En Combinación Con Arterias Hepáticas Aberrantes. RevArgAnatOnl 3: 18-21.

Pidal G, Oribe G, Arrayago I, Curra-Gagliano F, D'Anna E, Martín E. 2014. Vasculatura Portal Intrahepática: Descripción Anatómica, Radiológica y Ecográfica. Int J Morphol 32: 909-13. (Doi: 10.4067/S0717-95022014000300026)

Pinal D, Nuno C, Gonzalez M, Ibarra T. 2018. The Celiac Trunk and Its Anatomical Variations: A Cadaveric Study. J ClinMed Res 10: 321-29. (Doi:10.14740/jocmr3356w)

Rodríguez D, Fallas J. 2011. Variante anatómica: origen de la arteria hepática derecha de la arteria mesentérica superior. MedLeg Costa Rica 28: 71-74.

Saiz R, Ferrer M, Vázquez J, Falco E, Escudero
M, Vicens J, González M. 2017. Variantes según la clasificación de Michels y ramas extrahepáticas. Revisión del tema y descripción de hallazgos angiográficos en 99 pacientes tratados con quimioembolización. Intervencionismo 17: 60-69.

Sitarz R, Berbecka M, Mielko J, RawiczPruszyński K, Staśkiewicz G, Maciejewski R, Polkowski W. 2018. Awareness of hepatic arterial variants is required in surgical oncology decision making strategy: Case report and review of literature. Oncol Lett 15: 6251-56. (Doi: 10.3892/ol.2018.8106)

Skórzewska $A$, Stajgis $P$, Grzymisławska $M$, Rojewska M, Krajecki M, Bruska M, Juszkat $R$. 2014. Rare variations of hepatic arteries in association with variable origin of gastroduodenal artery found in multidetector computed tomography angiography. Folia Morphol 73: 531-35.

Ursic M, Ravnik D, Hribernik M, Pecar J, Butinar J, Fazarinc G. 2007. Gross anatomy of the portal vein and hepatic artery ramifications in dogs: corrosion cast study. AnatHistolEmbryol 36: 83-87. (Doi: 10.1111/j.1439-0264.2006. 00719.x)

Wang Y, Cheng C, Wang L, Li R, Chen J, Gong $S$. 2014. Anatomical variations in the origins of the celiac axis and the superior mesenteric artery: MDCT angiographic findings and their probable embryological mechanisms. EurRadiol 24: 1777-84. (Doi:10.1007/s00330-014-3215-9) White R, Weir J, Sullivan C, Mustafa S, Yeap P, Budak M, Sudarshan T, Zealley I. 2015. The Celiac Axis Revisited: Anatomic Variants, Pathologic Features, and Implications for Modern Endovascular Management. Radio Graphics 35: 879-98. (Doi:10.1148/rg. 2015140243) 